\title{
Optical properties-microstructure-texture relationships of dried apple slices: spatially resolved diffuse reflectance spectroscopy as a novel technique for analysis and process control
}

\author{
Nghia Nguyen Do Trong $^{1 *}$, Anna Rizzolo ${ }^{2}$, Els Herremans ${ }^{1}$, Maristella Vanoli ${ }^{2,5}$, Giovanna \\ Cortellino $^{2}$, Chyngyz Erkinbaev ${ }^{1}$, Mizuki Tsuta ${ }^{3}$, Lorenzo Spinelli ${ }^{4}$, Davide Contini ${ }^{5}$, Alessandro \\ Torricelli ${ }^{5}$, Pieter Verboven ${ }^{1}$, Josse De Baerdemaeker ${ }^{1}$, Bart Nicolaii ${ }^{1}$, Wouter Saeys ${ }^{1}$ \\ ${ }^{1}$ BIOSYST-MeBioS, KU Leuven, Willem de Croylaan 42, 3001 Leuven, Belgium \\ ${ }^{2}$ Consiglio per la Ricerca e Sperimentazione in Agricoltura - Unità di ricerca per i processi \\ dell'industria agroalimentare (CRA-IAA), via Venezian 26, I 20133 I-Milan, Italy \\ ${ }^{3}$ National Food Research Institute, 2-1-12 Kan-nondai, Tsukuba, Ibaraki 305-8642, Japan \\ ${ }^{4}$ Istituto di Fotonica e Nanotecnologie, CNR, piazza Leonardo da Vinci 32, I 20133 Milan, Italy \\ ${ }^{5}$ Politecnico di Milano, Dipartimento di Fisica, piazza Leonardo da Vinci 32, I 20133 Milan, Italy \\ *Email: nghia.nguyendotrong@biw.kuleuven.be; ndtnghia@gmail.com
}

\section{Abstract}

The potential of spatially resolved diffuse reflectance spectroscopy in the 500-1000 nm range by means of a fiber-optics probe was investigated for acquiring scattering and absorption properties of air dried apple rings subjected to different pre-treatment conditions: without osmo-dehydration (TQ) and with osmo-dehydration for 1 (OSMO1) and 3 hours (OSMO2). The fresh apple rings were produced from 'Golden Delicious' apples at harvest $(\mathrm{H})$ and 5 month storage at 2 conditions: controlled atmosphere (CA) and normal atmosphere (NA). Microstructure properties of the dried apple rings were also obtained from X-ray micro-CT measurements. The TQ samples were found to have significantly higher scattering properties, thicker tissue, smaller pore sizes, were less crispy, and required higher snapping work or rupture energy than the OSMO1 and OSMO2 samples. On the other hand, no significant differences were observed between the scattering properties, microstructure, and textural quality of the OSMO1 and OSMO2 apple rings. From these results, it was concluded that there is a clear process-microstructure-quality relation in osmo-air-dried apples which can be measured non-destructively with spatially resolved diffuse reflectance spectroscopy. 
Therefore, this study confirmed the potential of spatially resolved diffuse reflectance spectroscopy for non-destructive quality assessment of air-dried apple slices, which provides perspectives for drying process optimization.

Keywords: spatially resolved spectroscopy, diffuse reflectance, scattering, absorption, osmo-airdried apple, texture

\section{Introduction}

Dried fruit is an important category of processed foods on the market with a worldwide annual production of $9.5 \times 10^{9} \mathrm{~kg}$ in 2012 , which is $13 \%$ higher than the production in 2011 (International Nut and Dried Fruit Council, 2013). It has been demonstrated that food consumers demand that dried food products are not only nutritious, but also texturally tasty and flavoursome (Szczesniak, 1971). Therefore, textural quality, which can be related to the food microstructure, is one of the key determining factors for food consumers' quality perception (Szczesniak and Kahn, 1971; Szczesniak, 1972; Szczesniak, 2002). This texture-microstructure relation has, for example, been reported for confectionary (Decker \& Ziegler, 2003), gelled systems (Borwankar, 1992; Barrangou, Drake, Daubert, \& Foegeding, 2006), dried apples (Acevedo, Briones, Buera, \& Aguilera, 2008), and aerated sugar gels (Herremans et al., 2013). In these works, microstructural or sensory properties of the studied food samples were clearly observed in good correlation with mechanical or rheological parameters usually considered as representing parameters for food texture quality. Besides its impact on the texture, food microstructure also has significant effects on the gas and heat transport in the food matrix and its nutritional, chemical, and microbiological stability (Aguilera, 2005; Mebatsion et al., 2008).

Several researchers have investigated the effects of processing conditions on the microstructure of final food samples as observed by means of light or electron microscopy. Some example cases are sweet potato patties (Walter \& Hoover, 1984), smoked Atlantic salmons 
53 (Sigurgisladottir, Ingvarsdottir, Torrissen, Cardinal, \& Hafsteinsson, 2000), high pressure processed

54 fresh carrots (Araya et al., 2007), and frozen strawberry tissues (Van Buggenhout, Grauwet, Van

55 Loey, \& Hendrickx, 2008). Although the microscopy techniques are very valuable, they are destructive and involve considerable sample preparation time, and complex and expensive equipment. As a result, these techniques are mainly used for academic purposes, but rarely in a food industrial environment (Aguilera, 2005). Therefore, the food industry is demanding a fast, nondestructive, and sufficiently accurate method for on-line or in-line food microstructure assessment which would allow optimizing the production processes to obtain a desired microstructure for the final food products.

Optical measurement techniques have high potential here thanks to the fast and highly sensitive interaction of the propagating photons with the microstructure and chemical composition of the analysed samples. Inhomogeneities in the microstructure of the real food systems introduce spatial inhomogeneities in the refractive index, which consequently change the trajectories of the propagating photons being known as scattering phenomena. In addition to this, the intensity of the light also decays as a result of absorption of the photons by the spectrally active food components. This absorption is specific in the sense that photons will only be absorbed when their energy matches with the energy needed to excite the molecular vibration state. Therefore, spectroscopic measurements are usually performed at multiple wavelengths or over a certain wavelength range. These two main phenomena, scattering and absorption, together determine the intensity of the post-interacting or outgoing light. In a clear liquid the concentration can be directly estimated from the intensity ratio of the outgoing and incoming light (transmittance) by Beer's law. However, in the case of turbid samples like food products this is not straightforward as transmittance measurements are very challenging and the intensity decay depends both on the scattering and absorption properties (Saeys, Velazco-Roa, Thennadil, Ramon, \& Nicolaï, 2008). 

quality attributes with some success in the last two decades thanks to the non-invasive interactions between the Vis/NIR light and the food matrices (Nicolaï et al., 2007; Huang, Yu, Xu, \& Ying, 2008). Since food samples are usually turbid media in which the interactions between the propagating light and food matrices are influenced by both scattering and absorption phenomena, multivariate calibration models have been used to relate the measured spectroscopic data to the real food quality attributes. Since these multivariate calibration models are generally based on Beer's law the effects of scattering phenomena on the post-interacting light should be reduced or minimized to improve the performance of the constructed calibration models (Martens \& Næs, 1991). Consequently, conventional Vis/NIR spectroscopy usually discards scattering or microstructure information and has limited success in evaluating the quality attributes relating to food microstructure (Nicolaï et al., 2007). In this regard, novel or improved optical measurement techniques are essentially required for food microstructure assessments.

As new approaches in biomedical research, multiple Vis/NIR spectroscopy measurements of the post-interacting light at difference spatial locations (spatially or space-resolved) or at various temporal incidents (time-resolved) have been extensively studied to separate the scattering and absorption information of the measured samples. As these measurements are typically done at multiple wavelengths, these methodologies are known as spatially-resolved reflectance spectroscopy (SRS) and time-resolved reflectance spectroscopy (TRS). Both techniques have been elaborated and identified as promising techniques in biomedical research for detection of abnormalities in human tissues related to changes in the microstructure and/or chemical composition (Tuchin, 2007; Tuchin, 2008).

In the domain of food and agricultural products, TRS has been investigated for non-destructive evaluation of microstructural information or microstructure-relating quality attributes of fruit such as apples, pears, and nectarines (Cubeddu et al., 2001; Zerbini et al., 2002; Zerbini et al., 2006; 
Nicolaï et al., 2008; Rizzolo et al., 2009). However, the high complexity and cost of the current TRS equipment hampers its uptake by the food industry. With SRS, measurements based on one moving optical fiber, multi-fiber probes, a hyperspectral camera or even a camera imaging at a single wavelength have been explored to assess microstructure-related attributes of meat, fruit and vegetables (Tu, Jancsók, Nicolaï, \& De Baerdemaeker, 2000; Qin \& Lu, 2008; Xia, Weaver, Gerrard, \& Yao, 2008; Qin, Lu, \& Peng, 2009; Romano, Argyropoulos, Nagle, Khan, \& Müller, 2012; Itoh, De Baerdemaeker, Nguyen Do Trong, Wang, \& Saeys, 2013; Nguyen Do Trong et al., 2013). However, in these researches the effect of processing conditions on the final microstructure of the samples was not taken into account.

Recently, Herremans et al. (2013) demonstrated the potential of SRS for monitoring the effect of processing conditions on the microstructure, and related texture properties, of sugar foams. These promising results suggest that SRS could also be a useful technique for monitoring microstructure changes in more complex food matrices like fresh and dried fruit. Therefore, the potential of fiberprobe SRS for detecting microstructure differences in dried apple rings caused by different processing conditions has been investigated in this study.

\section{Materials and methods}

\subsection{Dried apple ring samples}

\section{'Golden Delicious' apples}

One hundred fifty six 'Golden Delicious' apples were harvested at commercial maturity from an orchard in Laimburg (Bolzano province, Italy) and were transported to the laboratory in Milan, Italy. These apples were then sorted in ascending maturity according to descending values of absorption coefficient $\mu_{a}$ of chlorophyll at $670 \mathrm{~nm}$ measured by a TRS setup (Torricelli et al., 2008). The ranked apples were randomly distributed into 9 groups. This random selection aimed at creating groups of apples with almost the same distribution of maturity levels. Three groups, labelled as $\mathrm{H}$ groups, were processed and analysed directly after harvest. Each of these groups was given one of the 3 pre- 
treatment conditions (without osmotic dehydration and with osmotic dehydration for 1 or 3 hours) followed by air drying. Three other groups, noted as CA, were stored for 5 months in controlled atmosphere $\left(+1{ }^{\circ} \mathrm{C}, 2 \mathrm{kPa} \mathrm{O}{ }_{2}+0.7 \mathrm{kPa} \mathrm{CO}_{2}\right)$. The remaining 3 groups, noted as $\mathrm{NA}$, were also kept at $+1{ }^{\circ} \mathrm{C}$ under normal atmosphere for 5 months. After the storage period, the CA and NA groups were taken out of the storage place, equilibrated their internal temperature and subjected to the same pre-treatments (with and without osmotic dehydration) and air drying as the $\mathrm{H}$ groups. Before pretreatment, each apple fruit was cored and cut into a ring of $5 \mathrm{~mm}$ thickness for further use (pretreatment and drying).

\section{Pre-treatments of the apple rings}

Pre-treatment of the apple rings was carried out by osmo-dehydration at 3 levels: without osmodehydration (noted as TQ), with osmo-dehydration $\left(20^{\circ} \mathrm{C}\right.$, atmospheric pressure) in sucrose solution (60\% w/w) for 1 hour (noted as OSMO1) and 3 hours (noted as OSMO2). During osmo-dehydration, the ring of each fruit was packed in a tulle bag and immersed in the sugar solution. The sucrose solution was re-circulated at a flow rate of $1.5 \mathrm{~L} / \mathrm{min}$ by a pump. The ratio fruit : solution was 1:3.

After osmo-dehydration, the apple rings were taken out of the sucrose solution, drained, rinsed gently with water and covered by adsorbent paper for a few minutes to remove excess water. These osmo-dehydrated rings (OSMO1 and OSMO2) and the rings without osmo-dehydration treatment (TQ) were all subjected to air drying.

\section{Air drying}

The apple rings (OSMO1, OSMO2, and TQ) were dried by air flow $\left(80{ }^{\circ} \mathrm{C}\right.$ dry bulb, atmospheric pressure, $1.5 \mathrm{~m} / \mathrm{s}$ speed) in a pilot alternate upward-downward air circulated drier until constant weight: when the difference in weight of the ring was less than $1 \mathrm{mg} / \mathrm{g}$ solids after 90 additional minutes of drying. 

H-OSMO2), CA storage (CA-TQ, CA-OSMO1, CA-OSMO2), and NA storage (NA-TQ, NA-OSMO1, NAOSMO2). The dried apple ring produced from each fresh apple after pre-treatment and air drying was first kept at CRA-IAA, Milan, Italy for destructive quality assessment, and then it was carefully packed in a plastic bag and sent to KU Leuven, Belgium for spatially resolved diffuse reflectance and $\mathrm{X}$-ray micro-CT measurements.

\subsection{SRS Setup}

\subsubsection{Setup description}

The setup for SRS measurements in the range $500-1000 \mathrm{~nm}$ used in this research is the same as the one described by Nguyen Do Trong et al. (2013) and Herremans et al. (2013), and is schematically illustrated in Figure 1. It basically consisted of a halogen light source, a fiber-optics sensing probe integrating one illumination fiber and five detection fibers, a spectrograph, a camera, and a computer with a data acquisition device. In brief, Vis/NIR light from an AvaLight-DHc halogen lamp (Avantes, Eerbeek, The Netherlands) was delivered through an optical fiber to illuminate the sample. Five detection fibers collected diffuse reflected light at different source-detector distances ranging from 0.3 to $1.2 \mathrm{~mm}$. All the fibers (Thorlabs, USA) having a core diameter of $200 \mu \mathrm{m}$ were all integrated inside a stainless steel sensing probe. The five detection fibers guided the diffuse reflected light into a spectrograph (Horiba Jobin-Yvon, New Jersey, USA) which splitted the light from each of these fibers into its spectral components and projected these onto a Hamamatsu C7041 CCD camera (Hamamatsu, Louvain-La-Neuve, Belgium). Controlling of the SRS setup and data acquisition were performed by a LabView program (LabView 8.5, National Instruments, Texas, USA) developed in the lab. 


\subsubsection{Setup calibration and measurement}

Wavelength calibration linked each pixel on the spectral dimension of the CCD camera to its corresponding waveband and was described in detail by Herremans et al. (2013). The relative diffuse reflectance spectra of the detection fibers from a measured sample were obtained as the ratios of the dark-corrected intensities acquired for that sample to the dark-corrected intensities collected in an integrating sphere (50 mm diameter, Avantes, Eerbeek, The Netherlands). Thanks to the highly diffuse reflectance of the sphere (reflecting more than $98 \%$ lights in the range $400-1000 \mathrm{~nm}$ ) the measured signals were compensated for dark noises, variations in the light source intensity, differences in efficiencies of different pixels of the camera and differences in efficiencies of the detection fibers.

\subsubsection{Estimation of optical properties from SRS measurements}

Thirty six optical liquid phantoms with known optical properties were prepared to calibrate the SRS measurements and to validate the procedure for estimation of optical properties. These liquid phantoms were aqueous mixtures of intralipid 20\% (Fresenius Kabi, Sweden) serving as scatterers and Indian ink (Chartpak Inc., USA) acting as absorber in the 500-1000 nm range. Optical properties of these phantoms were determined as reported in Nguyen Do Trong et al. (2012) and covered a wide range of optical properties for food and agricultural products $\left(\mu_{a}=0.001-2.6 \mathrm{~cm}^{-1} ; \mu_{\mathrm{s}}{ }^{\prime}=5.7-\right.$ $135.3 \mathrm{~cm}^{-1}$ ). A metamodelling method (Couckuyt, Forrester, Gorissen, De Turck, \& Dhaene, 2012) was then developed to relate the measured SRS data of the phantoms to the corresponding reference optical properties. In this approach, Stochastic Kriging was used to build a metamodel relating the acquired SRS profiles for the liquid phantoms (output) to their reference optical properties (input). Five calibration metamodels were constructed, one for each of the five detection fibers.

Kriging is a popular technique to approximate deterministic noise-free data. It has been originally conceived as a geostatistical estimator that estimates the value for an unobserved location, by using 
samples from different locations surrounding it (e.g. the elevation, z, of a landscape as a function of the geographic location). Kriging is based on the idea that the value at an unknown point should be the average of the known values at its neighbours - weighted by the neighbours' distance to the unknown point (Sacks, Welch, Mitchell, \& Wynn, 1989). While the interpolation properties of the Kriging methodology are advantageous for many deterministic simulation problems, it could produce undesirable results when dealing with stochastic simulations. Stochastic Kriging (Staum, 2009) has, therefore, been suggested as an extension of Kriging for approximation instead of interpolation for stochastic phenomena.

The metamodel construction was implemented in the ooDACE toolbox (Couckuyt, Forrester, Gorissen, De Turck, \& Dhaene, 2012) which is a versatile Matlab toolbox that implements Stochastic Kriging. For each liquid phantom, SRS measurement had been repeated 9 times to provide estimations of the measurement noise as a function of the optical properties. The toolbox used the mean diffuse reflectance values of the detection fibers and the intrinsic covariance matrix, representing the variance of the output values. Initially, a first set of Kriging models was constructed using an initial set of candidate features. These candidates were then ranked using a Bayesian variable selection method. In the next step, a new promising feature was incorporated into the Kriging model, after which the new Kriging model was scored according to the leave-one-out-crossvalidation prediction error. This process was repeated until the accuracy of the Kriging model stopped improving (Couckuyt, Forrester, Gorissen, De Turck, \& Dhaene, 2012) and the final metamodel was acquired.

To estimate optical properties of a new sample in inverse procedure, a Nelder-Mead optimization algorithm was employed to iteratively estimate the optical properties of a sample by matching the spatially resolved diffuse reflectance profiles simulated with the metamodel to the acquired ones. All these calculations were performed in Matlab (version 7.10, The MathWorks Inc., Natick, USA). 

left out of the phantom set and the remaining 35 phantoms were used for constructing the calibration metamodel. These acquired calibration metamodels were then employed to estimate the optical properties of the validation phantom by using its measured spatially resolved diffuse reflectance profiles in the inverse estimation procedure. The accuracy of the optical properties estimation was then evaluated by comparing the estimated optical properties for this validation phantom with the reference optical properties. In this study, 16 phantoms covering the range of optical properties expected for the dried apples were selected and used one by one in the 16 validation steps. The estimation errors for the 16 validation phantoms were then combined to quantify the average prediction performance for the absorption coefficient $\mu_{a}$ and reduced scattering coefficient $\mu_{s}^{\prime}$. The average prediction error was calculated by taking the root of the mean of the squared differences between the predicted and actual optical properties for all the validation phantoms, noted as RMSECV (Root Mean Squared Error of Cross-Validation). were averaged and the resulting SRS data were used to determine the optical properties of that ring. The wavelength region from 590 to $1000 \mathrm{~nm}$ which had high signal-to-noise light intensities was used in the further analyses (reflectance calculation, optical properties estimation). 
The microstructure of selected dried apple samples was imaged by means of X-ray micro-CT. The most contrasting pre-treatments for drying within NA samples (NA-TQ and NA-OSMO2) were selected for the experiment. Within each group, samples of 4 fruit rings were scanned. Using a cork borer, small cylindrical samples ( $3 \mathrm{~mm}$ diameter) were excised from the dried apple, approximately 5 $\mathrm{mm}$ from the peel, excluding regions in which vascular tissue could be discerned visually. The thickness of the apple slices was not altered when preparing the samples. The samples were mounted on the rotating holder and stabilized using parafilm. X-ray micro-CT measurements were performed on a SkyScan 1172 system (Bruker microCT, Kontich, Belgium), operated at $55 \mathrm{keV}$ source voltage and $181 \mu \mathrm{A}$ current and with an isotropic image pixel resolution of $2.44 \mu \mathrm{m}$. The samples were rotated in $0.35^{\circ}$ steps over a total of $180^{\circ}$, each time averaging 3 frames to acquire a radiographic image of 1048 by 2000 pixels. The time to obtain all images for the 3D scan was 29 minutes per sample. The projection images were loaded into dedicated software (NRecon1.6.3.2, Bruker microCT, Kontich, Belgium) to reconstruct virtual cross-sections of the sample. This resulted in a 3D greyscale data stack, digitized to 880 slices of 2000 by 2000 pixels. The images were smoothed by a Gaussian smoothing kernel, and corrected for rings and beam hardening, which are common artefacts in X-ray CT images. For image analysis a cylindrical volume of interest (diameter $2.5 \mathrm{~mm}$ ) was cropped centrally in the imaged volume to exclude interference with the excised borders of the sample. The remaining volume for analysis measured $5.4 \mathrm{~mm}^{3}$. The images were filtered in 3D space using a median filter with filter radius of 2 pixels. Otsu's algorithm (Otsu, 1979) was applied for binarizing the image by separating two peaks in the greyscale frequency distribution: pixels with lower intensities than the Otsu threshold were assigned to the background (air) and pixels with a higher intensity than the threshold were assigned to the apple tissue material. Individual 3D objects smaller than 27 voxels were considered to be noise and were filtered out of the datastack. Morphometric parameters describing the microstructure were calculated on the 3D data using CTAn v.1.12.0.0 (Bruker microCT, Kontich, Belgium). Porosity was calculated as the fraction of 
segmented air pixels to the total amount of pixels in the analysed dataset. For the analysis of pore and (dehydrated) tissue thicknesses, the corresponding binary images were thinned until a skeletonized representation of the medial axes was obtained (Hildebrand \& Ruegsegger, 1997). Next, a sphere was fitted on every pixel of the skeleton until the boundary of the material was reached. The diameters of the fitted spheres give information on the local thicknesses of the microstructural features.

\subsection{Destructive quality assessment}

\section{Textural quality measurements}

Textural quality attributes of the dried apple rings were measured by a TA-XT plus Texture Analyser (Stable Micro Systems, UK) through bending-snapping tests fitted with a $50 \mathrm{~N}$ load cell and using the HDP/3PB Three Point Bending Rig. The lower supporting blades were separated at a distance of $45 \mathrm{~mm}$, and the compressing blade was driven down between the two supports at a speed of $0.17 \mathrm{~mm} / \mathrm{s}$, bending each apple ring until it snapped. From the force/displacement curve, the following parameters were acquired for that apple ring:

- Hardness (N): maximum force at break

- Total Area (N.mm or $\mathrm{mJ}$ ): work required to snap the ring (Snapping Work or Rupture Energy)

- Crispness Index (MPa): computed according to the following formula (Farris, Gobbi, Torreggiani, \& Piergiovanni, 2008):

$$
\text { Crispness Index }=\frac{8 \times L^{\mathrm{B}} \times\left(X_{H}-X_{L}\right)}{6 \times \Delta L \times \pi \times\left(d_{0}^{4}-d_{i}^{4}\right)}
$$

Where:
$L(m):$
support separation 

modulus determination and $\Delta L$ flexure between $X_{H}$ and $X_{L}$

$$
d_{o}(m): \quad \text { ring outer diameter }
$$

300

$d_{i}(m):$

ring inner diameter

\section{Browning Index (BI)}

302

303

304

305

306

308

309

The colour of each dried apple ring was measured at 2 opposite sides by means of a CM-2600D colorimeter (Konica Minolta Inc., Japan) using the primary illuminant D65 and $10^{\circ}$ observer in the $\mathrm{L}^{*} \mathrm{a}{ }^{*} \mathrm{~b}$ colour space. Acquired values of $L, a$, and $b$ were averaged for each ring.

The Browning Index (BI) indicates the purity of brown colour of the dried apple ring and was computed according to the following formula (Askari, Emam-Djomeh, \& Mousavi, 2008):

$$
B I=\frac{100(x-0.31)}{0.17}
$$

Where:

$$
x=\frac{a+1.75 L}{5.645 L+a-3.012 b}
$$

\section{Statistical Analysis}

Two-way analysis of variance (ANOVA) was implemented to investigate the effects of processing and storage conditions on the quality attributes of the dried apple samples. Apart from processing also storage conditions and the interaction between both were significant. The differences of the quality attributes among sample groups of the same storage conditions at $95 \%$ significance level was established by using one-way analysis of variance and Tukey's honest multiple comparisons by means of SAS Enterprise Guide (SAS Institute Inc., USA). 


\section{Results and discussion}

\subsection{Dried apple quality attributes}

The average values and standard deviations of the quality parameters (Hardness, Crispness, Snapping Work, and Browning Index) of the dried apple rings as measured with the reference methods are presented in Table 1.

\section{Position of Table 1}

It can be seen from Table 1, that at all storage conditions ( $\mathrm{H}, \mathrm{CA}$, and NA) the apple rings which had been subjected to osmo-dehydration prior to the air-drying are crispier, as indicated by the higher Crispness Index, than the ones without osmo-dehydration. The crispness is highest for the OSMO2 rings which had been subjected to a longer osmo-dehydration time. Moreover, the TQ rings (without osmo-dehydration) required significantly larger Snapping Work or efforts for mastication as compared to the OSMO1 and OSMO2 rings. On the other hand, only the difference in Hardness among the $\mathrm{H}-\mathrm{TQ}$ and $\mathrm{H}-\mathrm{OSMO} 2$ groups was significant. The Browning Index was significantly higher for the TQ rings obtained from NA and CA storages, which indicates that the apple rings without osmo-dehydration had a darker brown colour than the ones with osmo-dehydration.

In general, the texture quality and appearance (colour) of the dried apple rings which had been subjected to osmo-dehydration (OSMO1 and OSMO2) can be considered better than that of the rings without osmo-dehydration (TQ). On average, the OSMO2 samples, which had been subjected longer to osmo-dehydration ( $3 \mathrm{~h}$ vs. $1 \mathrm{~h}$ ) were crispier than the OSMO1 samples. 
The X-ray CT images illustrated in Figure 2 offer a detailed insight in the 3D microstructure of the scanned samples. The dried apple samples clearly have a highly porous structure as a large number of irregular black pores is present in between the complex tissue network.

\section{Position of Figure 2}

The representative morphometric parameters (porosity, dehydrated tissue and pore thicknesses) describing the apple ring microstructure calculated on the 3D data are presented in Table 2.

As can be seen from Table 2, the pre-treatment of the apple slices by osmo-dehydration prior to air drying resulted in significantly different microstructural characteristics for the dried apple samples. The porosity of the NA-OSMO2 samples is significantly higher than the porosity of the NATQ ones. This is also reflected in the different average thicknesses of the tissue as well. The tissues of TQ samples are also thicker than those of OSMO2 samples. Average pore space diameters (pore thicknesses) are significantly larger for OSMO2 samples as compared to those for TQ samples.

\subsection{SRS}


In Figure 3, the $z$-axis is the relative reflectance calculated by using an integrating sphere as a reference as described in section 2.2.2. Fiber 1 is the closest detection fiber and fiber 5 is the furthest one from the illumination point. The diffuse reflectance spectrum of each detection fiber shows an increasing trend in the beginning which flattens out at higher wavelengths. This is due to the absorption of the colour pigments in the apple rings as evidenced visually by their colour appearance or Browning Index. The small valley at $670 \mathrm{~nm}$ which can be seen in several spectra indicates the presence of chlorophyll in the dried apple flesh. At each wavelength, the diffuse reflectance values decrease with increasing source-detector distance or fiber position. This can be explained by the fact that light exiting the sample at a larger distance from the incident light beam has travelled a longer path through the sample and thus has had more chance to be absorbed or scattered. In comparison to fresh apple tissue (Nguyen Do Trong, et al., 2013), there is no clear absorption peak of water at $970 \mathrm{~nm}$. This could have been expected as there is only very little water left in the tissue after air drying until constant weight.

\subsection{Validation of the optical properties estimation on liquid phantoms} coefficient $\mu_{s}{ }^{\prime}$ ) of the 16 validation phantoms are plotted against their reference values. 

0.978 , respectively for the absorption coefficient $\mu_{a}$ and reduced scattering coefficient $\mu_{s}{ }^{\prime}$ values.

Moreover, the mean prediction errors RMSECV (Root Mean Squared Error of Cross-Validation) are approximately $0.1 \mathrm{~cm}^{-1}$ for $\mu_{a}$ and $3.9 \mathrm{~cm}^{-1}$ for $\mu_{s}^{\prime}$. In the wavelength range below $80 \mathrm{~cm}^{-1}$, the predicted reduced scattering coefficient $\mu_{s}{ }^{\prime}$ are quite close to the measured ones. Beyond $80 \mathrm{~cm}^{-1}$, some deviations can be observed. A possible explanation for these deviations could be that the scattering in the liquid phantoms with higher intralipid concentrations is no longer independent. In this study we considered a linear relation between the intralipid concentration and the scattering coefficient values, but it has been shown that this relation may become nonlinear for higher intralipid concentrations due to dependent scattering (Zaccanti, Del Bianco, \& Martelli, 2003). Further research would be needed to confirm this hypothesis. However, the prediction errors are sufficiently small as compared to the average reference values of $\mu_{a}\left(1.0 \mathrm{~cm}^{-1}\right)$ and $\mu_{s}{ }^{\prime}\left(38.7 \mathrm{~cm}^{-1}\right)$ in the validation set to conclude that this approach is useful for deriving the optical properties of the dried apple rings from the acquired spatially resolved diffuse reflectance spectra.

\subsection{Optical properties of dried apples}

The estimated optical properties (absorption coefficient $\mu_{a}$ and reduced scattering coefficient $\mu_{s}{ }^{\prime}$ ) spectra of the apple rings are illustrated in Figure 5.

\section{Position of Figure 5}

As can be seen from Figure 5 , the reduced scattering coefficients $\mu_{s}{ }^{\prime}$ of the TQ apple rings in the 700-1000 nm range are significantly higher than those of the OSMO1 and OSMO2 groups under the considered confidence intervals for all the storage conditions ( $H, C A$ and NA). Although the reduced 
scattering coefficient $\mu_{s}{ }^{\prime}$ values for the OSMO1 group in this range are clearly higher than those for the OSMO2 group, this difference was not significant. All the scattering profiles exhibit a decreasing trend with increasing wavelength in the $590-700 \mathrm{~nm}$ range. It should be noted here that the estimated scattering information in the $590-700 \mathrm{~nm}$ range may be less reliable than in the $700-1000$ $\mathrm{nm}$ range as the prediction error of the inverse metamodelling approach presented in Figure 4 (b) was higher for the higher scattering values. For this reason, the further interpretation of the scattering information and comparison to the microstructure and textural quality attributes of the apple rings was limited to the $700-1000 \mathrm{~nm}$ range. $700 \mathrm{~nm}$ after which they remain quite constant and are of small values (Figure 5). For all samples slight deviations can be observed at $670 \mathrm{~nm}$, indicating the presence of chlorophyll. The higher absorption values before $670 \mathrm{~nm}$ could be related to the formation of chromophores (e.g., melanoidins through Maillard reactions) during the air drying process. These observations are in good agreement with the appearance colour of the apple rings and the acquired SRS profiles as observed in Figure 3 . The absorption coefficient $\mu_{a}$ values before $700 \mathrm{~nm}$ of TQ apple rings which had not been pre-treated by osmo-dehydration are significantly higher than those of the OSMO1 and OSMO2 groups pre-treated by osmo-dehydration under the considered confidence intervals (data not shown). These are also reflected in the significant higher Browning Index (BI) values of the TQ apple rings as compared to the OSMO1 and OSMO2 rings (Table 1). The observed differences in $\mu_{a}$ values between the OSMO1 and OSMO2 groups were not significant.

\subsection{Optical properties-microstructure-texture relations}

Further investigation of the scattering information, microstructure, and textural quality attributes (Hardness, Crispness Index, and Snapping Work) reveals some interesting relations among them. The apple rings which had not been pre-treated by osmo-dehydration prior to air drying (TQ group) had less porosity, thicker tissue, and smaller pore sizes than the ones with osmo-dehydration (OSMO2 
group) (Table 2), which consequently made them less crispy and required more rupture energy during mastication than the osmo-dehydrated apple rings (Table 1). However, the osmo-dehydrated apple rings still had comparable Hardness as compared to the ones without osmo-dehydration, which would give positive contributions to the consumers' perception during the chewing process. Because of the thicker tissue and smaller pore sizes (Table 2), the TQ apple rings consequently had more interfaces resulting in a refractive index mismatch (e.g., air pore-tissue, cell-cell regions within the tissues, intercellular medium-cell compartments...) than the OSMO2 rings. This resulted in higher scattering coefficient values $\mu_{s}{ }^{\prime}$ for the TQ groups as compared to the OSMO2 groups (Figure 5). A clear relation between process conditions, microstructure and textural quality has thus been observed, which is consequently reflected in the scattering coefficient values obtained with SRS.

\section{Conclusions}

Fresh apple rings from 'Golden Delicious' apples at harvest $(\mathrm{H})$ and after five months of storage under controlled atmosphere (CA) and normal atmosphere (NA) conditions were subjected to 3 different pre-treatments: without osmo-dehydration (TQ) and with osmo-dehydration for 1 hour (OSMO1) and 3 hours (OSMO2). All the pre-treated apple rings were then air dried until constant weight. Diffuse reflectance measurements by SRS in the $500-1000 \mathrm{~nm}$ range, microstructure measurements by X-ray micro-CT technique, and destructive reference measurements for texture and appearance quality attributes were carried out on these samples. The estimated optical properties (reduced scattering coefficient $\mu_{s}{ }^{\prime}$ and absorption coefficient $\mu_{a}$ ) of the air dried apple rings from SRS measurements exhibited strong relations with the microstructural information and the quality parameters from destructive measurements. For all storage conditions $(H, C A$, and $N A)$, the TQ apple rings (without osmo-dehydration) had higher scattering, thicker tissue, smaller pore sizes, were less crispy, and required larger snapping efforts than the OSMO1 and OSMO2 apple rings

451 (with osmo-dehydration). Moreover, the TQ ring samples also had higher absorption coefficient $\mu_{a}$ than the osmo-air-dried ones (OSMO1 and OSMO2), in agreement with their higher Browning Index. 

of the OSMO1 and OSMO2 apple rings were, however, not significant.

It can thus be concluded that there is a clear relation between processing conditions and microstructure and textural quality in dried apples, which is consequently reflected in the scattering coefficient values obtained with SRS. As this technique is fast, non-destructive and does not require sample preparation, it has potential for on-line monitoring of the microstructure of dried apple rings and more general for drying process optimization.

\section{Acknowledgements} FP7-226783 - InsideFood). The opinions expressed in this document do by no means reflect the official opinion of the European Union or its representatives. The aids from Professor Depeursinge and his co-workers at the Swiss Federal Institute of Technology (EPFL, Lausanne, Switzerland) in developing the fiber-optic SRS setup are gratefully acknowledged. The provision of the ooDACE toolbox from the Department of Information Technology (INTEC), Ghent University, Belgium is gratefully acknowledged. Nghia Nguyen Do Trong is a PhD student funded by the Interfaculty Council for Development Cooperation, KU Leuven (IRO scholarship). Mizuki Tsuta would like to acknowledge the Japanese Society for the Promotion of Science (JSPS) for funding as a JSPS Postdoctoral Researcher for Research Abroad. We would also like to acknowledge the Hercules Foundation and

471 the Institute for the Promotion of Innovation through Science and Technology (IWT-Vlaanderen, 472 doctoral scholarship 093469). Wouter Saeys has been funded as a Postdoctoral Fellow of the 473 Research Foundation - Flanders (FWO). 
Acevedo, N. C., Briones, V., Buera, P., \& Aguilera, J. M. (2008). Microstructure affects the rate of chemical, physical and color changes during storage of dried apple discs. Journal of Food Engineering, 858, $222-231$.

Aguilera, J. M. (2005). Why food microstructure? Journal of Food Engineering, 67, 3- 11.

Araya, X. I. T., Hendrickx, M., Verlinden, B. E., Van Buggenhout, S., Smale, N. J., Stewart, C., \& John Mawson, A. (2007). Understanding texture changes of high pressure processed fresh carrots: A microstructural and biochemical approach. Journal of Food Engineering, 80 (3), 873 - 884.

Askari, G. R., Emam-Djomeh, Z., \& Mousavi, S.M., (2008). Investigation of the effect of microwave treatment on the optical properties of apple slices during drying. Drying Technology, 26, 1362-1368.

Barrangou, L. M., Drake, M.A., Daubert, C.R., \& Foegeding, E.A. (2006). Sensory texture related to large-strain rheological properties of agar/glycerol gels as a model food. Journal of Texture Studies, 37 (3), $241-262$.

Borwankar, R.P. (1992). Food texture and rheology: A tutorial review. Journal of Food Engineering, 488 16,1 - 16.

Couckuyt, I., Forrester, A., Gorissen, D., De Turck, F., \& Dhaene, T. (2012). Blind Kriging: Implementation and performance analysis. Advances in Engineering Software, 49, 1-13.

Cubeddu, R., D’Andrea, C., Pifferi, A., Taroni, P., Torricelli, A., Valentini, G., Dover, C., Johnson, D., Ruiz-Altisent, M., \& Valero, C. (2001). Nondestructive quantification of chemical and physical properties of fruits by time-resolved reflectance spectroscopy in the wavelength range 650-1100 nm. Applied Optics, 40 (4), 538-543.

Decker, N. R., \& Ziegler, G. R. (2003). Mechanical properties of aerated confectionery. Journal of 496 Texture Studies, 34, 437-448.

Farris, S., Gobbi, S., Torreggiani, D., \& Piergiovanni, L. (2008). Assessment of two different rapid compression tests for the evaluation of texture differences in osmo-air-dried apple rings. Journal of Food Engineering, 88, 484-491.

500 Herremans, E., Bongaers, E., Estrade, P., Gondek, E., Hertog, M., Jakubczyk, E., Nguyen Do Trong, N., 501 Rizzolo, A., Saeys, W., Spinelli, L., Torricelli, A., Vanoli, M., Verboven, P., \& Nicolaï, B. (2013). 502 Microstructure-texture relationships of aerated sugar gels: novel measurement techniques for 503 analysis and control. Innovative Food Science and Emerging Technologies, 18, 202-211. thickness in three dimensional images. Journal of Microscopy, 185, 67-75. 
Huang, H., Yu, H., Xu, H., \& Ying, Y. (2008). Near infrared spectroscopy for on/in-line monitoring of quality in foods and beverages: A review. Journal of Food Engineering, 87 (3), 303-313.

International Nut and Dried Fruit Council, 2013: https://www.nutfruit.org/en/inc-global-statisticalreview-reflects-the-continued-increase-of-the-global-consumption-of-nuts-and-dried-fruits_70983

Itoh, H., De Baerdemaeker, J., Nguyen Do Trong, N., Wang, L., \& Saeys, W. (2013). Estimation of pear ripeness by hyperspectral laser scatter imaging. Proceedings of the 2013 IFAC Bio-Robotics. 27-29 March 2013. Osaka, Japan.

Martens, H., \& Næs, T. (1991). Multivariate Calibration. Wiley. ISBN: 978-0-471-93047-1.

Mebatsion, H., Verboven, P., Ho, Q., Verlinden, B., \& Nicolaï, B. (2008). Modelling fruit (micro)structures, why and how? Trends in Food Science \& Technology, 19 (2), 59-66.

Nguyen Do Trong, N., Watté, R., Aernouts, B., Verhoelst, E., Tsuta, M., Jakubczyk, E., Gondek, E., Verboven, P., Nicolaï, B., \& Saeys, W. (2012). Differentiation of microstructures of sugar foams by means of spatially resolved spectroscopy. Proceedings of SPIE, Volume 8439: Paper 843914.

Nguyen Do Trong, N., Erkinbaev, C., Tsuta, M., De Baerdemaeker, J., Nicolaï, B., \& Saeys, W. (2013). Spatially resolved spectroscopy for nondestructive quality measurements of Braeburn apples cultivated in sub-fertilization condition. Proceedings of SPIE, Volume 8881, Paper $88810 \mathrm{~L}$

Nicolaï, B. M., Beullens, K., Bobelyn, E., Peirs, A., Saeys, W., Theron, K. I., \& Lammertyn, J. (2007). Nondestructive measurement of fruit and vegetable quality by means of NIR spectroscopy: A review. Postharvest Biology and Technology, 46 (2), 99-118.

Nicolaï, B. M., Verlinden, B. E., Desmet, M., Saevels, S., Saeys, W., Theron, K., Cubeddu, R., Pifferi, A., \& Torricelli, A. (2008). Time-resolved and continuous wave NIR reflectance spectroscopy to predict soluble solids content and firmness of pear. Postharvest Biology and Technology, 47, 68-74.

Otsu, N. (1979). A threshold selection method from gray-level histograms. IEEE Transactions on systems, man, and cybernetics, 9, 62-66.

Qin, J., \& Lu, R. (2008). Measurement of the optical properties of fruits and vegetables using spatially resolved hyperspectral diffuse reflectance imaging technique. Postharvest Biology and Technology, 49, 355-365.

Qin, J., Lu, R., \& Peng, Y., (2009). Prediction of apple internal quality using spectral absorption and scattering properties. Transactions of the ASABE, 52 (2), 499-507.

Rizzolo, A., Vanoli, M., Zerbini, P. E., Jacob, S., Torricelli, A., Spinelli, L., Schouten, R. E., \& Tijskens, L. M. M. (2009). Prediction ability of firmness decay models of nectarines based on the biological shift factor measured by time-resolved reflectance spectroscopy. Postharvest Biology and Technology, 54, 131-140. 
Romano, G., Argyropoulos, D., Nagle, M., Khan, M. T., \& Müller, J. (2012). Combination of digital images and laser light to predict moisture content and color of bell pepper simultaneously during drying. Journal of Food Engineering, 109 (3), 438 - 448.

Sacks, J., Welch, W. J., Mitchell, T., \& Wynn, H. P. (1989). Design and analysis of computer experiments. Statistical Science, 4 (4), 409-435.

Saeys, W., Velazco-Roa, M. A., Thennadil, S. N., Ramon, H., \& Nicolaï, B. M. (2008). Optical properties of apple skin and flesh in the wavelength range from 350 to $2200 \mathrm{~nm}$. Applied Optics, 47 (7), $908-$ 919.

Sigurgisladottir, S., Ingvarsdottir, H., Torrissen, O. J., Cardinal, M., \& Hafsteinsson, H. (2000). Effects of freezing/thawing on the microstructure and the texture of smoked Atlantic salmon (Salmo salar). Food Research International, 33 (10), $857-865$.

Staum, J. (2009). Better simulation metamodeling: The why, what, and how of stochastic Kriging. Proceedings of the Winter Simulation Conference 2009, 119-133.

Szczesniak, A. S., (1971). Consumer awareness of texture and of other food attributes II. Journal of texture studies, 2 (2), 196- 206.

Szczesniak, A. S., \& Kahn, E. L. (1971). Consumer awareness of and attitudes to food texture I: Adults. Journal of texture studies, 2 (3), $280-295$.

Szczesniak, A. S., (1972). Consumer awareness of and attitudes to food texture II: Children and Teenagers. Journal of texture studies, 3 (2), $206-217$.

Szczesniak, A. S., (2002). Texture is a sensory property. Food Quality and Preference, 13 (4), 215 225.

Torricelli, A., Spinelli, L., Contini, D., Vanoli, M., Rizzolo, A., \& Eccher Zerbini, P. (2008). Time-resolved reflectance spectroscopy for nondestructive assessment of food quality. Sensing and Instrumentation for Food Quality and Safety, 2, 82-89.

Tu, K., Jancsók, P., Nicolaï, B., \& De Baerdemaeker, J. (2000). Use of laser-scattering imaging to study tomato-fruit quality in relation to acoustic and compression measurements. International Journal of Food Science \& Technology, 35 (5), $503-510$.

Tuchin, V. (2007). Tissue Optics - Light scattering methods and instruments for medical diagnosis. (2nd ed.). SPIE Press, Bellingham, Washington, USA.

Tuchin, V. (2008). Handbook of optical sensing of glucose in biological fluids and tissues. CRC Press.

Van Buggenhout, S., Grauwet, T., Van Loey, A., \& Hendrickx, M. (2008). Structure/processing relation of vacuum infused strawberry tissue frozen under different conditions. European Food Research and Technology, 226 (3), $437-448$. 
572 Walter JR., W. M. \& Hoover, M. W. (1984). Effect of pre-processing storage conditions on the

573 composition, microstructure, and acceptance of sweet potato patties. Journal of Food Science, 49

574 (5), $1258-1261$.

575 Xia, J., Weaver, A., Gerrard, D. E., \& Yao, G., (2008). Distribution of optical scattering properties of 576 four beef muscles. Sensing and Instrumentation for Food Quality and Safety, 2, 75-81.

577 Zaccanti, G., Del Bianco, S., \& Martelli, F., (2003). Measurements of optical properties of high-density 578 media. Applied Optics, 42 (19), $4023-4030$.

579 Zerbini, P. E., Grassi, M., Cubeddu, R., Pifferi, A., \& Torricelli, A., (2002). Nondestructive detection of 580 brown heart in pears by time-resolved reflectance spectroscopy. Postharvest Biology and 581 Technology, 25, 87-97.

582 Zerbini, P. E., Vanoli, M., Grassi, M., Rizzolo, A., Fibiani, M., Cubeddu, R., Pifferi, A., Spinelli, L., \& 583 Torricelli, A., (2006). A model for the softening of nectarines based on sorting fruit at harvest by 584 time-resolved reflectance spectroscopy. Postharvest Biology and Technology, 39, 223-232. 
Table 1 . Reference quality attributes of the dried apple rings. Reported values are mean \pm standard deviation $(\mathrm{n}=$ 20 for $\mathrm{H}$ groups and $\mathrm{n}=16$ for $\mathrm{CA}$, NA groups). Different letters in each storage condition indicate significant difference at $p \leq 0.05$; symbol * indicates significant difference at $p \leq 0.1$.

\begin{tabular}{lllll}
\hline Sample & Hardness $(\mathrm{N})$ & Crispness Index $(\mathrm{MPa})$ & Snapping Work $(\mathrm{mJ})$ & Browning Index (BI) \\
\hline H-OSMO1 & $3.1 \pm 1.2^{\mathrm{a}, \mathrm{b}}$ & $61.8 \pm 53.9^{\mathrm{a}}$ & $1.4 \pm 1.0^{\mathrm{a}}$ & $62.6 \pm 8.6^{\mathrm{a}}$ \\
H-OSMO2 & $4.0 \pm 1.2^{\mathrm{b}}$ & $155.9 \pm 99.9^{\mathrm{b}}$ & $1.4 \pm 0.7^{\mathrm{a}}$ & $64.0 \pm 7.3^{\mathrm{a}, \mathrm{b}}$ \\
H-TQ & $2.9 \pm 1.3^{\mathrm{a}}$ & $20.1 \pm 13.6^{\mathrm{a}}$ & $3.8 \pm 1.7^{\mathrm{b}}$ & $68.8 \pm 4.6^{\mathrm{b}}$ \\
\hline CA-OSMO1 & $7.7 \pm 1.6$ & $375.8 \pm 179.9^{\mathrm{b}}$ & $3.3 \pm 2.0^{\mathrm{a}}$ & $66.9 \pm 7.8^{\mathrm{a}}$ \\
CA-OSMO2 & $8.5 \pm 2.2$ & $376.9 \pm 150.9^{\mathrm{b}}$ & $3.9 \pm 1.2^{\mathrm{a}}$ & $63.6 \pm 5.4^{\mathrm{a}}$ \\
CA-TQ & $7.4 \pm 2.3$ & $159.9 \pm 77.7^{\mathrm{a}}$ & $6.2 \pm 2.7^{\mathrm{b}}$ & $84.8 \pm 7.0^{\mathrm{b}}$ \\
\hline NA-OSMO1 & $7.5 \pm 1.8$ & $247.5 \pm 89.4^{\mathrm{b}}$ & $3.9 \pm 1.1^{\mathrm{a}}$ & $63.5 \pm 7.2^{\mathrm{a}}$ \\
NA-OSMO2 & $8.0 \pm 1.7$ & $297.6 \pm 111.7^{\mathrm{b}}$ & $3.8 \pm 1.2^{\mathrm{a}^{*}}$ & $61.1 \pm 7.7^{\mathrm{a}}$ \\
NA-TQ & $7.5 \pm 1.8$ & $127.2 \pm 53.9^{\mathrm{a}}$ & $5.0 \pm 1.8^{\mathrm{a}^{*}}$ & $73.4 \pm 5.9^{\mathrm{b}}$ \\
\hline
\end{tabular}

Table 2. Microstructural information of the dried apple rings (NA-OSMO2 and NA-TQ). Reported results are mean \pm standard deviation $(n=4)$. Different letters indicate significant difference at $p \leq 0.1$.

\begin{tabular}{cccc}
\hline Sample Group & Porosity $(\%)$ & Tissue thickness $(\mu \mathrm{m})$ & Pore thickness $(\mu \mathrm{m})$ \\
\hline NA-OSMO2 & $82.0 \pm 1.6^{\mathrm{b}}$ & $20.5 \pm 0.4^{\mathrm{a}}$ & $134.1 \pm 18.1^{\mathrm{b}}$ \\
NA-TQ & $77.8 \pm 1.1^{\mathrm{a}}$ & $23.3 \pm 1.1^{\mathrm{b}}$ & $101.9 \pm 6.2^{\mathrm{a}}$ \\
\hline
\end{tabular}




\section{List of figure captions}

Figure 1. Schematic illustration of the setup for SRS measurement

Figure 2. Illustration of X-ray CT cross-sectional images of two air dried apple rings: (a) NA-TQ and (b) NA-OSMO2. Scale bar at the right corner of each image indicates $250 \mu \mathrm{m}$.

Figure 3. SRS spectra of one H-TQ apple ring

Figure 4. Scatter plots of predicted versus measured optical properties of the 16 validation phantoms: (a): absorption coefficient $\mu_{a}$; (b): reduced scattering coefficient $\mu_{s}{ }^{\prime}$. The diagonal black line represents the perfect prediction line. RMSECV: Root Mean Squared Error of Cross-Validation.

Figure 5. Estimated optical properties of the dried apple rings: reduced scattering coefficient $\mu_{s}^{\prime}$ (left) and absorption coefficient $\mu_{a}$ (right). Mean values are plotted in the main graphs. In the inserts, thick curves indicate mean values; thin curves represent the mean \pm standard deviation. 


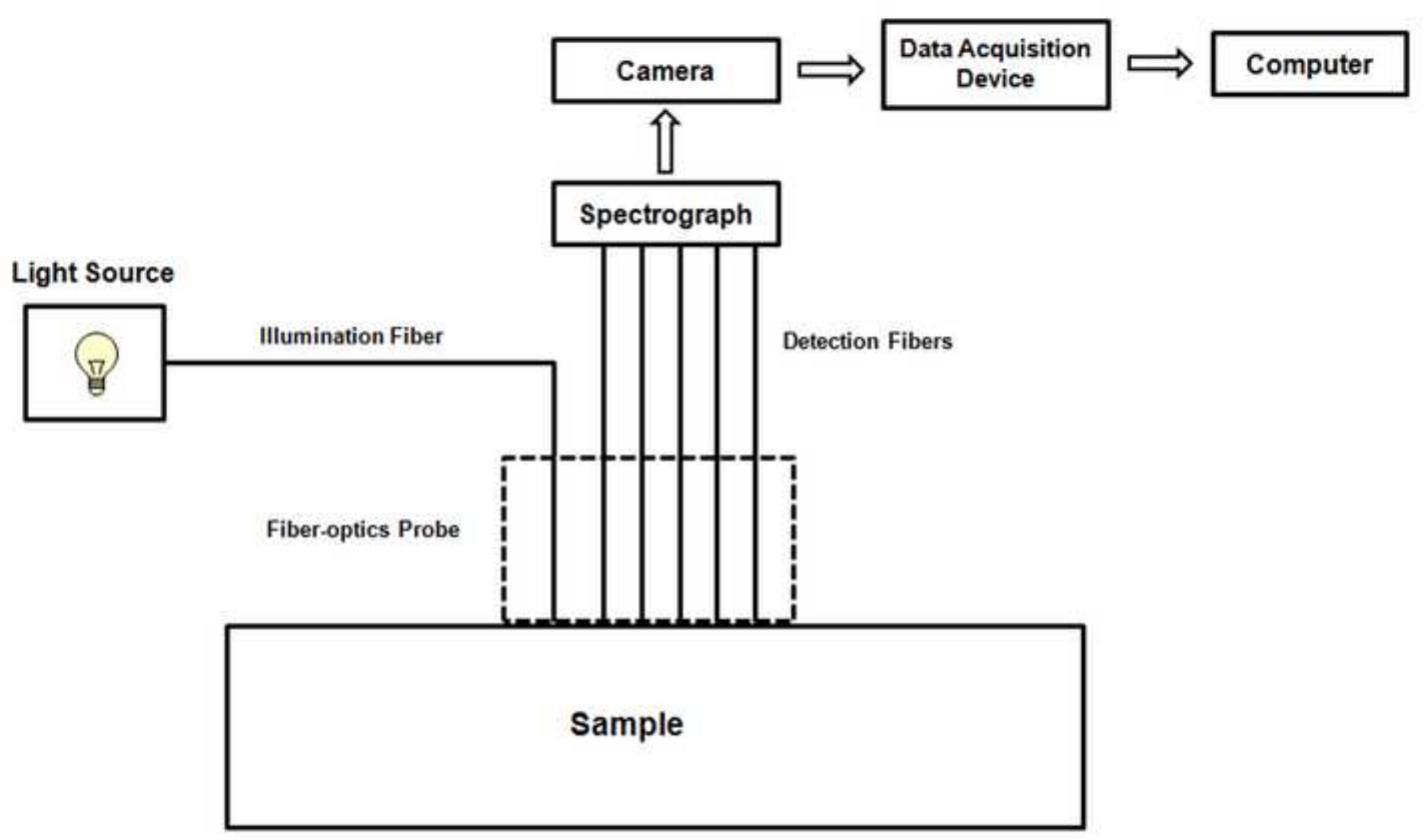

Light Source

Fiber-optics Probe

\section{Sample}




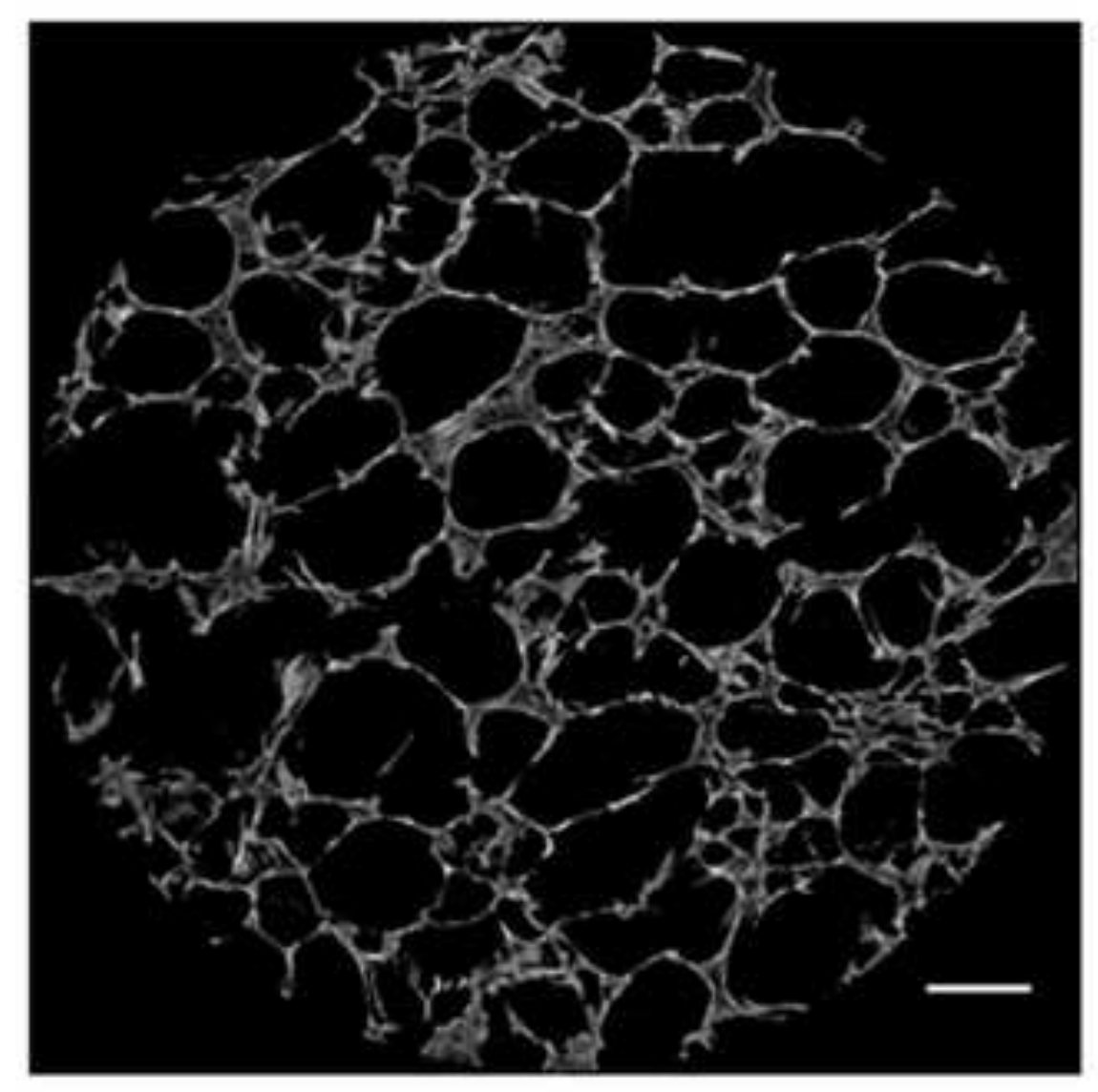

(a)

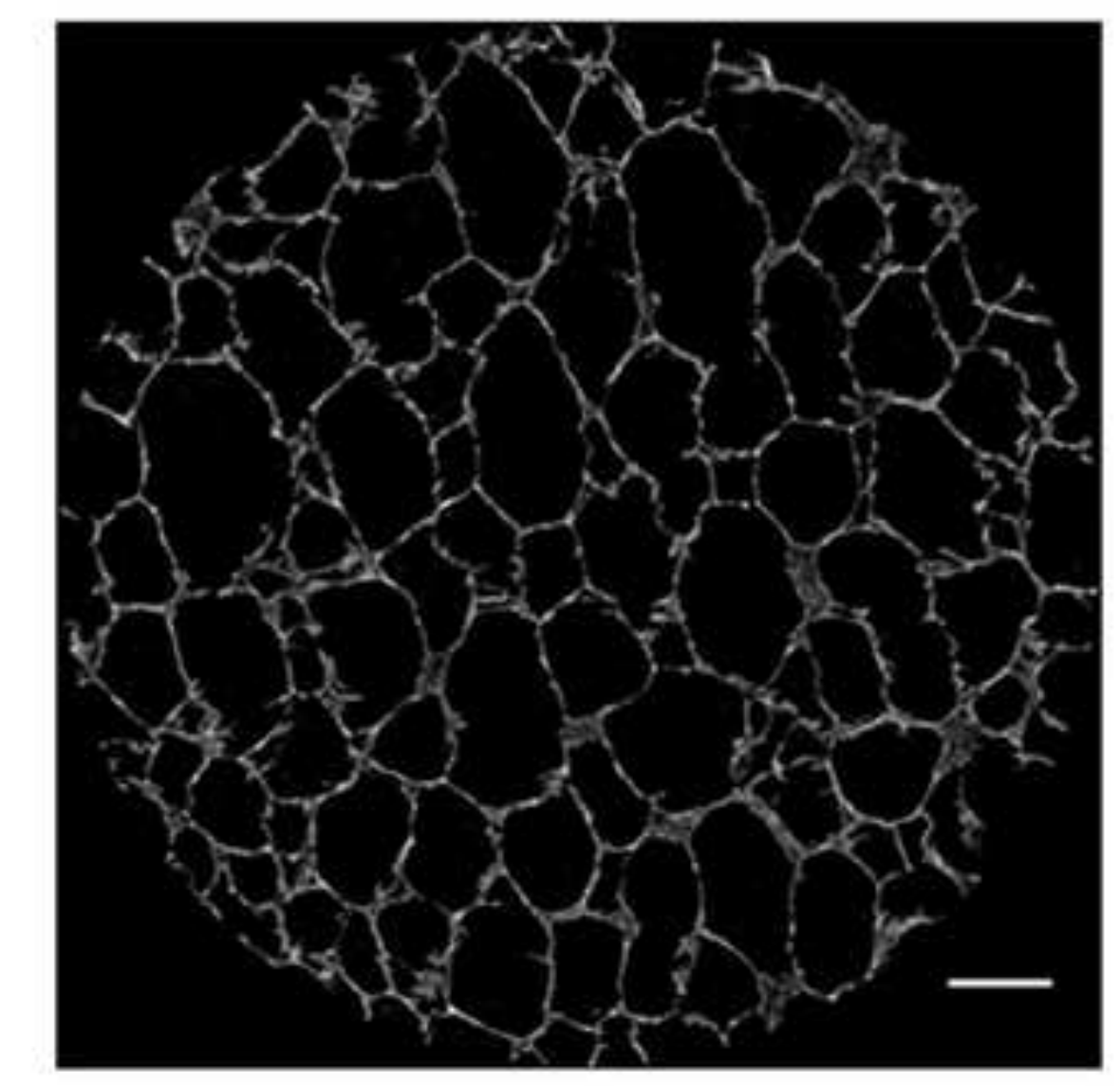

(b)

\section{Click here to download high resolution image}


Click here to download $\bar{h}$ igh resolution image

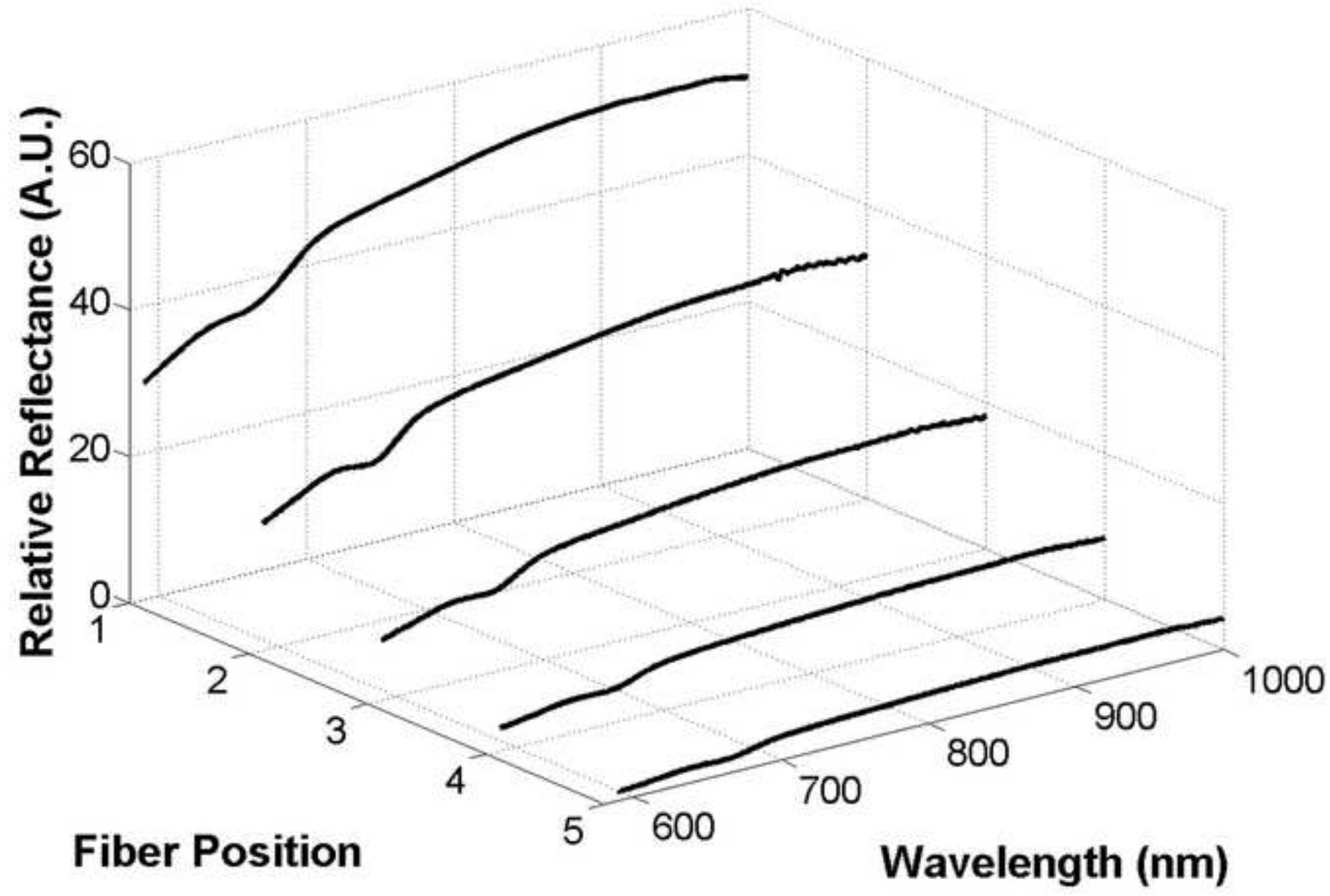




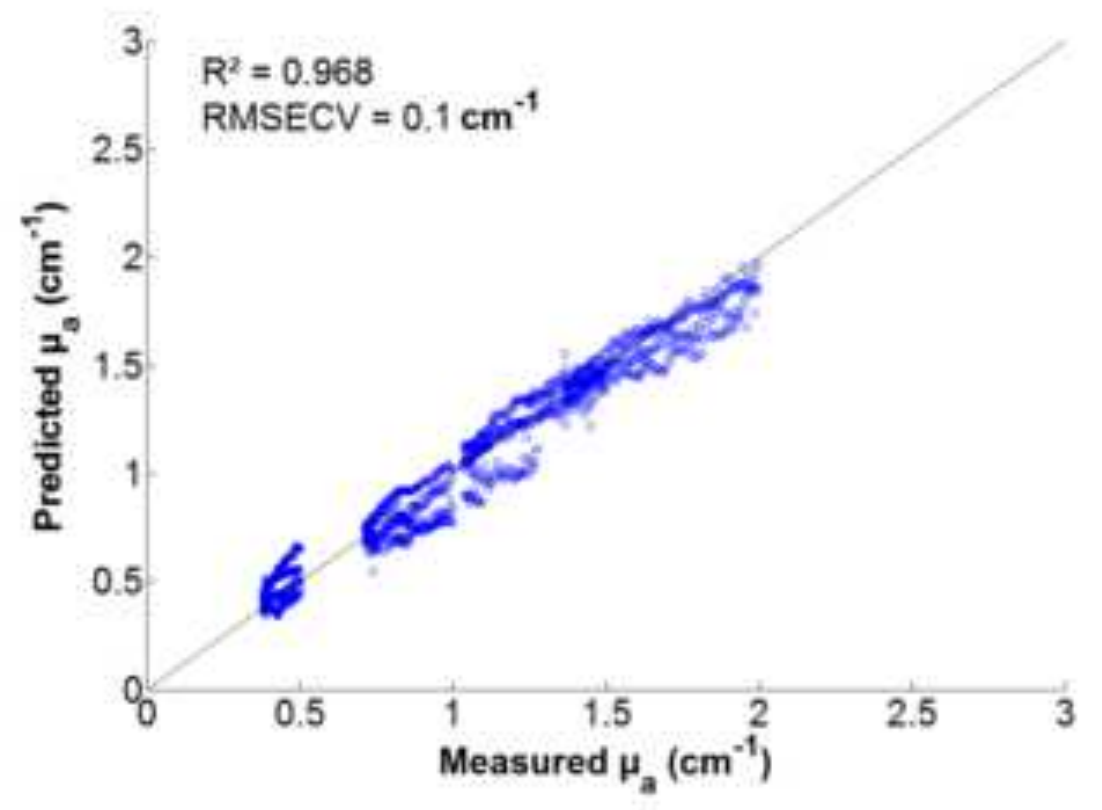

(a)

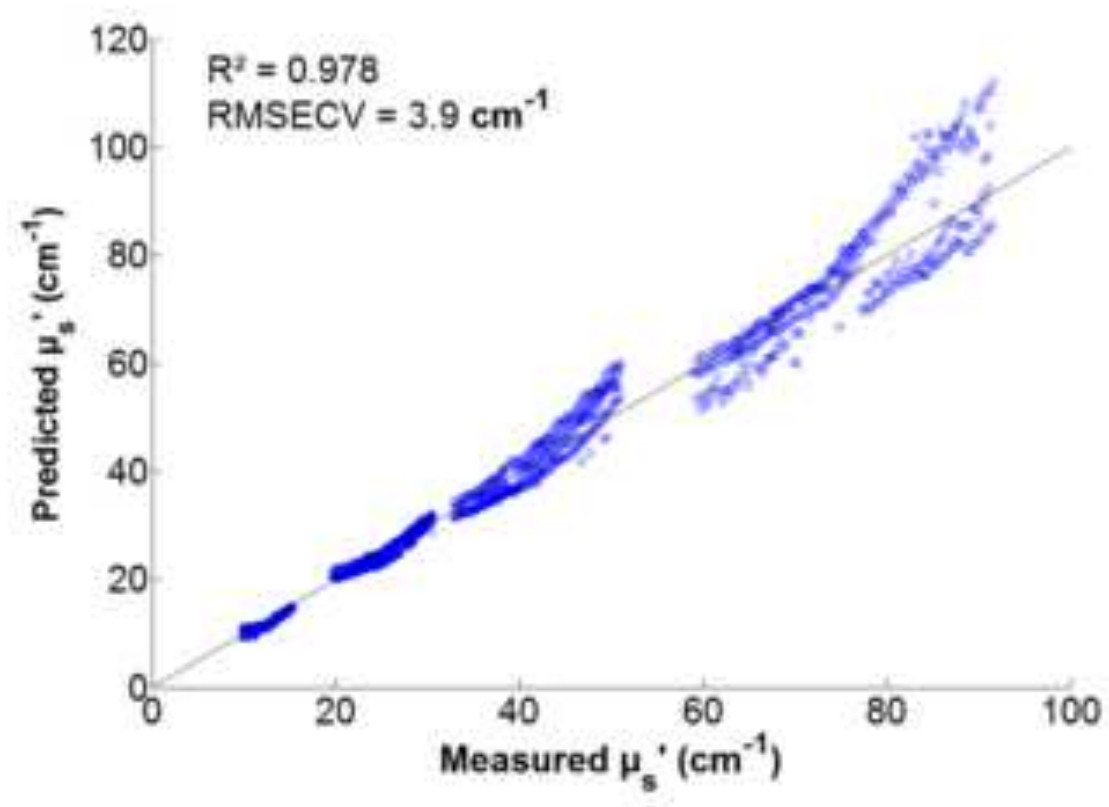

(b) 
Fig_5_Optical properties of dried apples Click here to download high resolution image
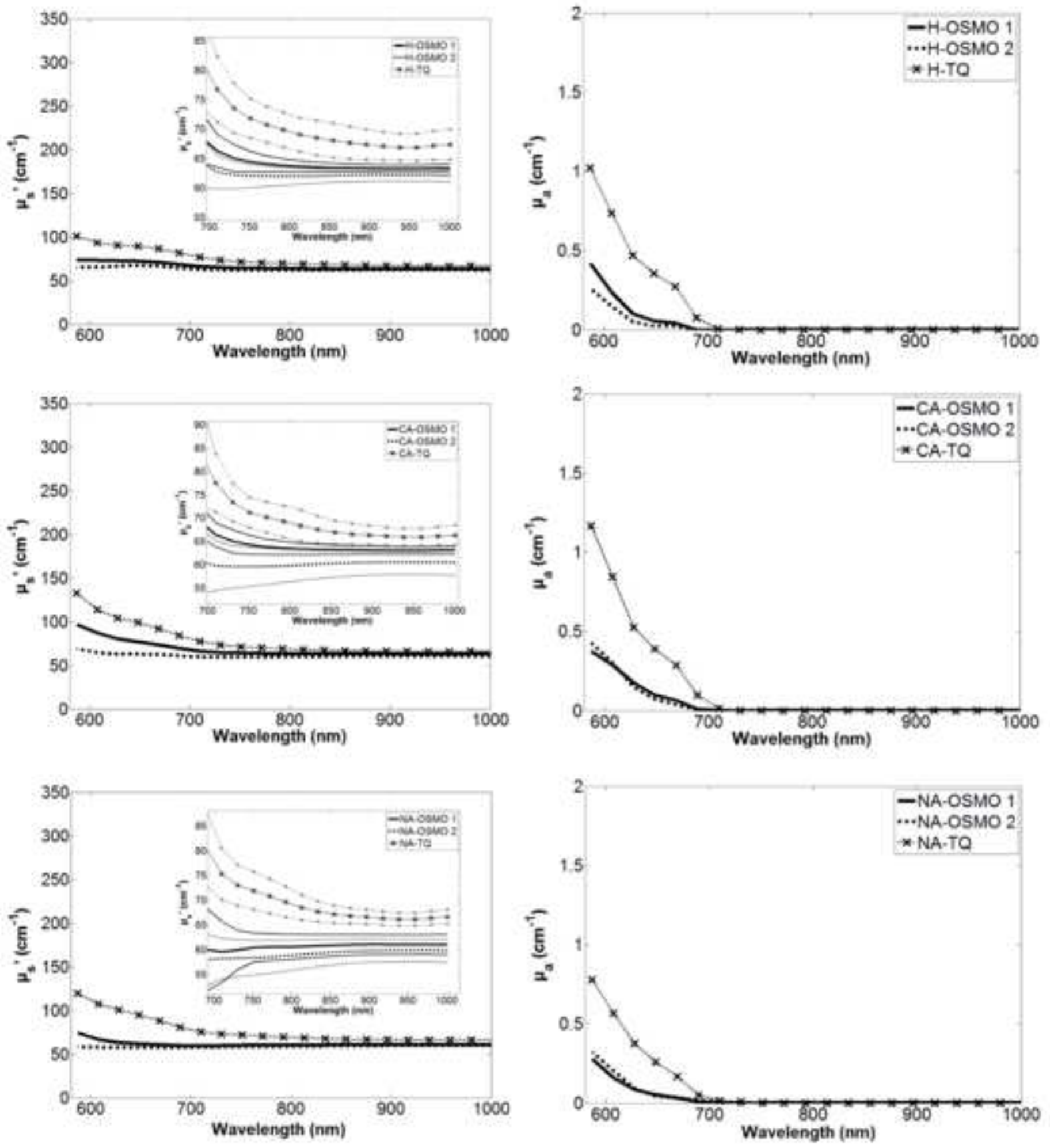